\title{
On Structural Stability for Semiflows
}

\author{
Juergen Quandt * \\ Division of Applied Mathematics, Brown University, Providence, RI, 02912, USA
}

\begin{abstract}
Some results on structural stability which are known to hold for flows of a compact manifold are extended to semiflows of a Banach space.
\end{abstract}

In this paper we consider the problem of structural stability for evolution equations which generate semiflows of Banach spaces. From a theoretical point of view it is advantageous in such a study to look at the semiflows directly without referring back to the generating equations. We will do this here by choosing a natural topology on the class of all semiflows of a given Banach space. We then ask the question under which conditions semiflows behave qualitatively alike if they are slightly perturbed in the above topology. Physically, this is motivated by the fact that, in experiments, semiflows can only be approximately determined.

Guided by the theory of flows, where the main assumption leading to structural stability is "hyperbolicity," we make the following observation. If we weaken both the concept of structural stability and hyperbolicity by restricting it to the global (bi-infinite) solutions, then the theory of flows carries over to the semiflow case. Physically, this may be justified by the fact that only those solutions seem to play a significant role in nature.

In Sects. 1 and 2 we extend results of Palis [6] and Pugh [7] on hyperbolic linear flows, and of Anosov [1] and Moser [5] on Anosov flows to the semiflow case, and Sect. 3 contains some ideas towards a general stability theorem for semiflows. A similar discussion for maps may be found in $[8,9]$.

\section{Introduction}

Let $(X,|\cdot|)$ be a Banach space. A $C^{r}$-semiflow of $X, r \geqq 0$, is a one-parameter family of $C^{r}$-maps $\{F(t): X \rightarrow X \mid t \geqq 0\}$ such that

1) $F(s+t)=F(s) F(t)$ for all $s, t \geqq 0$,

2) $F(0)$ is the identity,

3) $F(\cdot)(x):[0, \infty[\rightarrow X$ is continuous for every $x \in X$.

* Current address: Department of Mathematics, University of Central Florida, Orlando, FL 32816, USA 
The class of all $C^{r}$-semiflows of $X, r \geqq 0$, will be denoted by

$$
\operatorname{Sem}^{r}(X),
$$

and equipped with the quasimetric

$$
\sup \left\{\mid D^{i} F(t)(x)-D^{i} G(t)(x) \| t \in[0,1] \text { and } x \in X \text { and } i \in\{0, \ldots, r\}\right\}
$$

for $F(t), G(t) \in \operatorname{Sem}^{r}(X)$, where the value $\infty$ is allowed.

A global solution of a semiflow $F(t)$ is a function $v: \mathbb{R} \rightarrow X$ such that $F(t)(v(\tau))$ $=v(\tau+t)$ for all $t \geqq 0$ and $\tau \in \mathbb{R}$. Assumption 3$)$ above implies that any such function is continuous, i.e., an element of $C(\mathbb{R}, X)$.

Our first problem then is to find the right topology for $C(\mathbb{R}, X)$, i.e., to determine when two solutions should be considered close. The topology of uniform convergence would require the solutions to be close for all times $t$. This is certainly too strong. The topology of pointwise convergence requires the solutions to be close at finitely many times. This is quite satisfactory. We choose here the particular quasinorm

$$
\|v\|=\int_{-\infty}^{+\infty} e^{-|t|}|v(t)| d t
$$

for $v \in C(\mathbb{R}, X)($ see M. Komura [3]).

Since $C(\mathbb{R}, X)$ with this quasinorm is not complete, we may have to substitute its completion, whenever necessary.

Any continuous map $f: X \rightarrow X$ induces a map $\tilde{f}: C(\mathbb{R}, X) \rightarrow C(\mathbb{R}, X)$ through $\widetilde{f}(v)=f v$, where $f v$ means $f$ composed with $v$. For the time one map $F(1)$ of a semiflow $F(t)$ we simply write $F$, and $I(F)$ will denote an $\omega$-invariant set of $F(t)$, i.e., $F(t)[I(F)] \subseteq I(F)$ for all $t \geqq 0$. We then define

$$
\widetilde{I(F)}=\{v \in C(\mathbb{R}, I(F)) \mid F(v(t))=v(t+1) \text { for all } t \in \mathbb{R}\},
$$

that is the set of all global solutions of $F(t)$ with values in $I(F)$, and

$$
S(\widetilde{F})=\{v \in C(\mathbb{R}, X) \mid F(v(t))=v(t+1) \text { for all } t \in \mathbb{R}\},
$$

that is the set of global solutions of $F(t)$, and

$$
A(F)=\bigcap_{t \geqq 0} F(t)[X] .
$$

The following proposition contains several basic properties. In particular, it shows that $A(F)$ consists of all values in $X$ through which a global solution of $F(t)$ passes.

Let $p_{\tau}: C(\mathbb{R}, X) \rightarrow X, \tau \in \mathbb{R}$, denote the $\tau$-th projection.

Proposition 1. If $F(t)$ is a $C^{r}$-semiflow of $X, r \geqq 0$, then the diagram

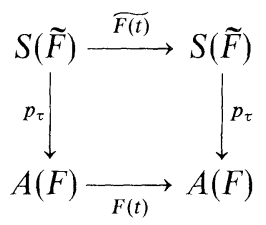


commutes for all $t \geqq 0$ and $\tau \in \mathbb{R}$ with $p_{\tau}[S(\tilde{F})]=A(F)$, and $\widetilde{F(t)}$ is bijective on $S(\widetilde{F})$ for every $t \geqq 0$.

Proof. Let $t \geqq 0, \tau \in \mathbb{R}$ and $v \in S(\widetilde{F})$. Then

$$
\begin{aligned}
p_{\tau}(\overline{F(t)}(v(\cdot))) & =p_{\tau}(F(t) v(\cdot))=p_{\tau}(v(\cdot+t))=v(\tau+t) \\
& =F(t) v(\cdot+\tau)=F(t)\left(p_{\tau}(v(\cdot))\right),
\end{aligned}
$$

and hence the above diagram commutes. To see that $p_{\tau}[S(\widetilde{F})]=A(F)$, let $v(\tau) \in p_{\tau}[S(\tilde{F})]$, i.e., $v \in S(\tilde{F})$ with $p_{\tau}(v)=v(\tau)$. For every $t \geqq 0$ we have $F(t)(v(\tau-t))$ $=v(\tau)$, and hence $v(\tau) \in A(F)$. Conversely, let $x \in A(F)$ and suppose there is no backward continuation to $-\infty$ for $x$, i.e., there exists a $t \in \mathbb{R}$ for which there is no $y \in X$ with $F(t)(y)=x$. But, in particular, $x \in F(t)[X]$, a contradiction. Finally, we fix $t \geqq 0$ and show that $\widetilde{F(t)}$ is bijective on $S(\widetilde{F})$. Let $v, w \in S(\widetilde{F})$ with $v \neq w$, i.e., there exists an $s \in \mathbb{R}$ such that $v(s) \neq w(s)$. Suppose that $\overline{F(t)}(v)=\overline{F(t)}(w)$. Then $v(s)=F(t)(v(s-t))$ $=F(t)(w(s-t))=w(s)$, a contradiction. Hence $\widetilde{F(t)}$ is one-to-one on $S(\widetilde{F})$. To see that it is also onto, let $v \in S(\widetilde{F})$, and observe that $\overparen{F(t)}(v(\cdot-t))=F(t) v(\cdot-t)=v$, with $v(\cdot-t) \in S(\widetilde{F})$.

Actually, it is quite easy to see that $\widetilde{F(t)}$ is a $C^{r}$-flow of $S(\widetilde{F})$. The fact that for every $t \geqq 0$, the map $\overline{F(t)}: C(\mathbb{R}, X) \rightarrow C(\mathbb{R}, X)$ is $C^{r}$, is a basic result from the theory of map spaces.

Let us now introduce a conjugacy between semiflows. We will say that two $C^{r}$-semiflows $F(t)$ and $G(t)$ of $X, r \geqq 0$, are inverse limit conjugate if there exists a homeomorphism $H: S(\widetilde{F}) \rightarrow S(\widetilde{G})$ which takes the global solutions of $F(t)$ onto the global solutions of $G(t)$ and preserves the orientation in time. This means that there exists a function $\alpha: S(\widetilde{F}) \rightarrow] 0, \infty[$ such that $H \overline{F(t)}(v)=\overline{G(\alpha(v) t)} H(v)$ for all $v \in S(\widetilde{F})$ and $t \in \mathbb{R}$. A $C^{r}$-semiflow $F(t)$ of $X, r \geqq 1$, is called $C^{r}$-inverse limit stable if there exists a $C^{r}$-neighborhood $U$ of $F(t)$ in $\operatorname{Sem}^{r}(X)$ such that for every $G(t) \in U$, the semiflows $F(t)$ and $G(t)$ are inverse limit conjugate. We want to emphasize that inverse limit stability compares global solutions, i.e., elements of $C(\mathbb{R}, X)$, rather than orbits, i.e., elements of $X$. Moreover, the concept of inverse limit conjugacy for semiflows is a natural extension of the concept of topological conjugacy for flows, i.e., for flows the two concepts coincide. Let us now ask which qualitative features are preserved under this form of stability. A global solution $v$ of a semiflow $F(t)$ is called an equilibrium solution if $F(t) v=v$ for all $t \in \mathbb{R}$, and a periodic solution if there exists an $\omega>0$ such that $F(t+\omega) v=v$ for all $t \in \mathbb{R}$. Such solutions will be preserved.

Proposition 2. Let $H: S(\widetilde{F}) \rightarrow S(\widetilde{G})$ and $\alpha: S(\widetilde{F}) \rightarrow] 0, \infty$ [ be an inverse limit conjugacy between two $C^{r}$-semiflows $F(t)$ and $G(t)$ of $X, r \geqq 0$. $G(t)$.

(i) If $v$ is an equilibrium solution of $F(t)$, then $H(v)$ is an equilibrium solution of

(ii) If $v$ is a periodic solution of $F(t)$, then $H(v)$ is a periodic solution of $G(t)$.

Proof. (i). Let $v$ be an equilibrium solution of $F(t)$. Then

$$
\widetilde{G(\alpha(v) t)} H(v)=H \widetilde{F(t)} H^{-1} H(v)=H \widetilde{F(t)}(v)=H(v) \text { for all } t \in \mathbb{R} .
$$


(ii). Let $v$ be a periodic solution of $F(t)$ with period $\omega$. Then

$$
\begin{gathered}
\widetilde{G(\alpha(v)(t+\omega))} H(v)=H \widetilde{F(t+\omega)} H^{-1} H(v)=H \widetilde{F(t+\omega)}(v)=H(v) \text { for } \\
\text { all } t \in \mathbb{R} . \quad \square
\end{gathered}
$$

Moreover, it is clear that the asymptotic behavior of global solutions will be preserved.

The following two technical lemmas are quite useful in proving inverse limit stability.

Lemma 1. Let $F(t), G(t)$ be two $C^{r}$-semiflows of $X, r \geqq 0$. If there exists a map $H: S(\widetilde{F}) \rightarrow C(\mathbb{R}, X)$ making the following diagram commute:

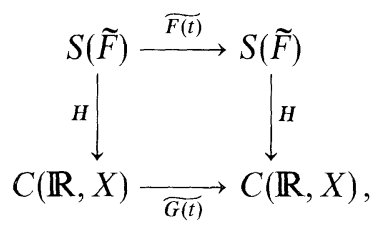

then there exists an "adjusted" map $\hat{H}: S(\widetilde{F}) \rightarrow C(\mathbb{R}, X)$ defined through $p_{s}(\hat{H}(v))$ $=p_{0}(H(v(\cdot+s)))$ for all $v \in S(\widetilde{F})$ and $s \in \mathbb{R}$, which also commutes the above diagram and, in addition, satisfies $\hat{H}[S(\widetilde{F})] \subseteq S(\widetilde{G})$. (This last condition is not automatically true for $H$ ).

Proof. Let $H$ and $\hat{H}$ be as above. Clearly the following diagram commutes for all $t \geqq 0$ :

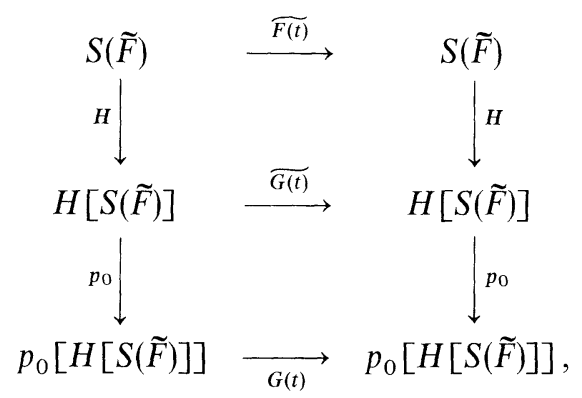

i.e., we have $G(t) p_{0} H=p_{0} H \widetilde{F(t)}$ for all $t \geqq 0$. We now must show that 1$)$ $\hat{H}[S(\widetilde{F})] \subseteq S(\widetilde{G})$ and 2) $\hat{H} \widetilde{F(t)}(v)=\widetilde{G(t)} \hat{H}(v)$ for all $v \in S(\widetilde{F})$ and $t \in \mathbb{R}$. We will use the fact that $\widetilde{F(t)}(v)=v(\cdot+t)$ for every $v \in S(\widetilde{F})$ and $t \in \mathbb{R}$.

Let $v \in S(\widetilde{F})$ and $s, t \in \mathbb{R}$. Then

Ad 1). $\quad G(1)\left(p_{t}(\hat{H}(v))\right)=G(1)\left(p_{0}(H(v(\cdot+t)))\right)$

$$
\begin{aligned}
& =G(1)\left(p_{0}(H(\widetilde{F(t)}(v)))\right) \\
& =p_{0}(H(\widetilde{F(1)}(\widetilde{F(t)}(v)))) \\
& =p_{0}(H(\widetilde{F(t+1)}(v))) \\
& =p_{0}(H(v(\cdot+t+1))) \\
& =p_{t+1}(\hat{H}(v)) .
\end{aligned}
$$

Ad 2). $\quad p_{s}(\hat{H}(\widetilde{F(t)}(v)))=p_{s}(\hat{H}(v(\cdot+t)))$

$$
=p_{0}(H(v(\cdot+t+s))) \text {, }
$$


and

$$
\begin{aligned}
\left.p_{s}(\widetilde{G(t)}(\hat{H}(v)))\right) & =G(t)\left(p_{s}(\hat{H}(v))\right) \\
& =G(t)\left(p_{0}(H(v(\cdot+s)))\right) \\
& =p_{0}(H(v(\cdot+t+s))) .
\end{aligned}
$$

Lemma 2. Let $H$ and $\hat{H}$ be as above.

(i) If $H$ is bounded by $\mu$, then $\hat{H}$ is bounded by $2 \mu$.

(ii) If $H$ is continuous, then so is $\hat{H}$.

Proof. Ad (i). Let $H$ be bounded by $\mu$, i.e., for all $v \in S(\widetilde{F})$

$$
\int_{-\infty}^{+\infty} e^{-|t|}\left|p_{t}(H(v))\right| d t \leqq \mu .
$$

Since with $v \in S(\widetilde{F})$ and $s \in \mathbb{R}$ also $v(\cdot+s) \in S(\widetilde{F})$, we have, in particular, that

$$
\left|p_{0}(H(v(\cdot+s)))\right| \leqq \mu .
$$

Hence

$$
\int_{-\infty}^{+\infty} e^{-|s|}\left|p_{s}(\hat{H}(v))\right| d s=\int_{-\infty}^{+\infty} e^{-|s|}\left|p_{0}(H(v(\cdot+s)))\right| d s \leqq 2 \mu .
$$

Ad (ii). Clear from (i).

Part (i) of this lemma shows that if $H$ is close to the identity [of $C(\mathbb{R}, X)]$, i.e., $H=\mathrm{id}+G$ with $G$ bounded (small), then so is $\hat{H}$.

\section{Hyperbolic Linear Semiflows}

Let $L(t)$ be a hyperbolic linear semiflow of $X$, i.e., $L(t)$ is continuous and the spectrum of $L=L(1)$ does not meet the unit circle. In this case the spectrum of $L(t)$ does not meet the unit circle for any $t>0$. Moreover, this is equivalent to saying that $L(t)$ has an exponential dichotomy, i.e., there exists a splitting of $X$ into $X^{s} \oplus X^{u}$, where $X^{s}, X^{u}$ are closed subsets of $X$ which are $\omega$-invariant under $L(t)$, and there are constants $M, N, v>0$ and an equivalent norm on $X$ such that

$$
\begin{aligned}
& |L(t)(x)| \leqq M e^{-v t}|x| \text { for all } x \in X^{s} \text { and } t \geqq 0 \text {, } \\
& |L(t)(x)| \geqq N e^{v t}|x| \quad \text { for all } \quad x \in X^{u} \text { and } t \geqq 0 \text {. }
\end{aligned}
$$

We set $L_{s}=L_{\mid X^{s}}, L_{u}=L_{\mid X^{u}}$ and $a=\max \left\{\left|L_{s}\right|,\left|L_{u}^{-1}\right|\right\}$, called the skewness of $L(t)$. (Hyperbolicity for linear maps is defined analogously.)

Let $F(t)$ be a Lipschitz semiflow of $X$, i.e., for every $t \geqq 0$ there exists a positive constant $M(t)$ such that

$$
|F(t)(x)-F(t)(y)| \leqq M(t)|x-y| \text { for all } x, y \in X .
$$

Suppose that $M(1)<1-a$ and that $F(t)$ is also bounded.

For a topological space $A$ we define

$$
C_{b}(A, C(\mathbb{R}, X))=\{f: A \rightarrow C(\mathbb{R}, X) \mid f \text { is continuous and bounded }\},
$$


with corresponding norm

$$
\|F\|_{b}=\sup \{\|F(v)\| v \in A\} .
$$

We begin with the following simple observation.

Lemma 3. Let $L(t)$ and $F(t)$ be as above. Then

(i) $\tilde{L(t)}$ is a hyperbolic semiflow of $C(\mathbb{R}, X)$,

(ii) $\widehat{F(t)}$ is a Lipschitz semiflow of $C(\mathbb{R}, X)$ with the same constants as $F(t)$, and bounded,

(iii) $\widetilde{L(t)}+\widetilde{F(t)}=\widetilde{L(t)+F(t)}$ for all suitable $t$.

\section{Proof. Clear.}

We can now prove the inverse limit conjugacy of $L(t)$ and $L(t)+F(t)$. In particular, both semiflows have homeomorphic sets of global solutions.

Theorem 1. Let $L(t)$ and $F(t)$ be as above. Then $L(t)$ and $L(t)+F(t)$ are inverse limit conjugate.

Proof. The proof follows Irwin [2, p. 114], and we begin with the following result for time one maps.

Let $F_{1}(t), F_{2}(t)$ be two Lipschitz semiflows of $X$ with constants at time one less than $1-a$ and bounded. Then there exists a unique map $G: S\left(\widetilde{L}+\widetilde{F_{1}}\right) \rightarrow C(\mathbb{R}, X)$ such that

$$
(\mathrm{id}+G)\left(\tilde{L}+\widetilde{F_{1}}\right)=\left(\tilde{L}+\widetilde{F_{2}}\right)(\mathrm{id}+G) \text { on } S\left(\tilde{L}+\widetilde{F_{1}}\right),
$$

where id denotes the identity on $C(\mathbb{R}, X)$, and id $+G: S\left(\widetilde{L}+\widetilde{F_{1}}\right) \rightarrow S\left(\tilde{L}+\widetilde{F_{2}}\right)$ is a homeomorphism.

Equation (1) is equivalent to

$$
\left(\tilde{L}+\widetilde{F_{2}}\right)(\mathrm{id}+G)\left(\tilde{L}+\widetilde{F_{1}}\right)^{-1}=\left(\tilde{L}+\widetilde{F_{1}}\right)\left(\tilde{L}+\widetilde{F_{1}}\right)^{-1}+G \quad \text { on } \quad S\left(\tilde{L}+\widetilde{F_{1}}\right) .
$$

We define

$$
\begin{aligned}
\mathscr{L}: C_{b}\left(S\left(\tilde{L}+\widetilde{F_{1}}\right), C(\mathbb{R}, X)\right) & \rightarrow C_{b}\left(S\left(\widetilde{L}+\widetilde{F_{1}}\right), C(\mathbb{R}, X)\right) \\
G & \mapsto \widetilde{L} G\left(\widetilde{L}+\widetilde{F_{1}}\right)^{-1},
\end{aligned}
$$

and

$$
\begin{aligned}
\mathscr{F}: C_{b}\left(S\left(\tilde{L}+\widetilde{F_{1}}\right), C(\mathbb{R}, X)\right) & \rightarrow C_{b}\left(S\left(\widetilde{L}+\widetilde{F_{1}}\right), C(\mathbb{R}, X)\right) \\
G & \mapsto \widetilde{F_{2}}(\mathrm{id}+G)\left(\widetilde{L}+\widetilde{F_{1}}\right)^{-1}-\widetilde{F_{1}}\left(\widetilde{L}+\widetilde{F_{1}}\right)^{-1} .
\end{aligned}
$$

Equation (2) is then equivalent to

$$
(\mathscr{L}+\mathscr{F})(G)=G,
$$

and $\mathscr{L}$ is hyperbolic with skewness $a$ and a corresponding splitting

$$
C_{b}\left(S\left(\tilde{L}+\widetilde{F_{1}}\right), C(\mathbb{R}, X)\right)=C_{b}\left(S\left(\tilde{L}+\widetilde{F_{1}}\right), C\left(\mathbb{R}, X^{s}\right)\right) \oplus C_{b}\left(S\left(\tilde{L}+\widetilde{F_{1}}\right), C\left(\mathbb{R}, X^{u}\right)\right) .
$$

Moreover, $\mathscr{F}$ is Lipschitz with a constant less than $1-a$, and therefore $\mathscr{L}+\mathscr{F}$ has a unique fixed point $G$. [Notice that the fixed point set of $\mathscr{L}+\mathscr{F}$ is equal to 
that of the contraction $(\mathrm{Id}-\mathscr{L})^{-1} \mathscr{F}$, where Id denotes the identity on $C_{b}\left(S\left(\widetilde{L}+\widetilde{F_{1}}\right), C(\mathbb{R}, X)\right)$ and $(\mathrm{Id}-\mathscr{L})^{-1}$ is the left inverse of Id $\left.-\mathscr{L}.\right]$

To see that the range of id $+G$ is contained in $S\left(\widetilde{L}+\widetilde{F_{2}}\right)$, we use Lemmas 1 and 2. They show that the range of id $+\hat{G}$ is contained in $S\left(\widetilde{L}+\widetilde{F_{2}}\right)$, that $\hat{G}$ satisfies Eq. (1) on $S\left(\widetilde{L}+\widetilde{F_{1}}\right)$, and that $\widehat{G} \in C_{b}\left(S\left(\widetilde{L}+\widetilde{F_{1}}\right), C(\mathbb{R}, X)\right)$. Uniqueness then implies that $\hat{G}=G$.

Reversing the roles of $F_{1}$ and $F_{2}$, one obtains a unique map $G^{\prime} \in C_{b}\left(S\left(\widetilde{L}+\widetilde{F_{2}}\right)\right.$, $C(\mathbb{R}, X)$ ) such that

$$
\left(\mathrm{id}+G^{\prime}\right)\left(\widetilde{L}+\widetilde{F_{2}}\right)=\left(\widetilde{L}+\widetilde{F_{1}}\right)\left(\mathrm{id}+G^{\prime}\right) \text { on } S\left(\widetilde{L}+\widetilde{F_{2}}\right),
$$

and it follows as above that the range of id $+G^{\prime}$ is contained in $S\left(\widetilde{L}+\widetilde{F_{1}}\right)$.

Equations (1) and (3) imply that

$$
\begin{aligned}
\left(\mathrm{id}+G^{\prime}\right)(\mathrm{id}+G)\left(\widetilde{L}+\widetilde{F_{1}}\right) & =\left(\mathrm{id}+\widetilde{G^{\prime}}\right)\left(\widetilde{L}+\widetilde{F_{2}}\right)(\mathrm{id}+G) \\
& =\left(\widetilde{L}+\widetilde{F_{1}}\right)\left(\mathrm{id}+G^{\prime}\right)(\mathrm{id}+G) \text { on } S\left(\widetilde{L}+\widetilde{F_{1}}\right) .
\end{aligned}
$$

One then sees that

$$
\left(\mathrm{id}+G^{\prime}\right)(\mathrm{id}+G)=\mathrm{id}+\left(G+G^{\prime}(\mathrm{id}+G)\right) \text { on } S\left(\widetilde{L}+\widetilde{F_{1}}\right),
$$

and since $G+G^{\prime}(\mathrm{id}+G)$ is continuous and bounded, it follows from uniqueness that

$$
\left(\mathrm{id}+G^{\prime}\right)(\mathrm{id}+G)=\mathrm{id} \quad \text { on } S\left(\widetilde{L}+\widetilde{F_{1}}\right),
$$

and analogously that

$$
(\mathrm{id}+G)\left(\mathrm{id}+G^{\prime}\right)=\mathrm{id} \quad \text { on } \quad S\left(\widetilde{L}+\widetilde{F_{2}}\right) .
$$

This shows that id $+G$ is a homeomorphism, since $G$ and $G^{\prime}$ are continuous.

We now set $F_{1} \equiv 0, F_{2} \equiv F$ and $H=\mathrm{id}+G$, and observe that for any $t \in \mathbb{R}$,

$$
\begin{aligned}
(\overline{L+F)(1)})(\overline{(L+F)(t)} H \overline{L(-t)}) & =\overline{(L+F)(t)}(\overline{L+F)(1)} H \overline{L(-t)} \\
& =\overline{(L+F)(t)} H \overline{L(1)} \overline{L(-t)} \\
& =\overline{(L+F)(t)} H \widetilde{L(-t))} L(1) \text { on } S(\widetilde{L}),
\end{aligned}
$$

and also that

$$
\begin{aligned}
\widetilde{(L+F)(t)} H \widetilde{L(-t)}-\mathrm{id}=(\overline{(L+F)(t)}-\widetilde{L(t))} H \widetilde{L(-t)} \\
+\widetilde{L(t)}(H-\mathrm{id}) \overline{L(-t)} \text { on } S(\widetilde{L}) .
\end{aligned}
$$

This shows that $\overline{(L+F)(t)} H \overline{L(-t)}-\mathrm{id}$ is continuous and bounded, so that by uniqueness

$$
H=\overline{(L+F)(t)} H \widetilde{L(-t)} \text { on } S(\tilde{L}),
$$

and the proof of the theorem is complete.

We notice that the above conjugacy is unique only if it is required to be within a finite distance of the identity.

Theorem 1 does not necessarily lead to a conjugacy on the phase space between $A(L)$ and $A(L+F)$. This is because neither the linear semiflow $L(t)$ nor the 
perturbed semiflow $(L+F)(t)$ need to be one-to-one on $A(L)$ and $A(L+F)$, respectively.

If there exist $r, s \in \mathbb{R}$ such that $L(\tau)[X]=L(r)[X]$ for all $\tau \geqq r$ and $\operatorname{ker}(L(\tau))$ $=\operatorname{ker}(L(s))$ for all $\tau \geqq s$, then $L(t)$ is one-to-one on $A(L)$ for all $t \geqq 0$. For example, if $X$ is finite dimensional, this condition is satisfied. Theorem 1 then yields a continuous, onto conjugacy between $A(L)$ and $A(L+F)$. If, in addition, $L+F$ is one-to-one on $A(L+F)$, then there exists a commuting homeomorphism between $A(L)$ and $A(L+F)$.

\section{Anosov Semiflows}

We now turn to the slightly more complicated situation of hyperbolic invariant sets for semiflows. A discussion in the context of semilinear parabolic equations may be found in [4] of R. Mané.

We will say that a $C^{r}$-semiflow $F(t)$ of $X, r \geqq 1$, has a (weak) hyperbolic structure for an $\omega$-invariant set $I(F)$ if for every $v \in \widetilde{I(F)}$ there exists a continuous splitting of $\bigcup_{t=-\infty}^{+\infty} T_{v(t)} X$ into a direct sum $X^{s} \oplus X^{0} \oplus X^{u}$, where

$$
\begin{gathered}
X^{i}=\bigcup_{t=-\infty}^{+\infty} X_{v(t)}^{i} \quad \text { for } \quad i=s, 0, u, \\
T_{v(t)} F(\tau)\left[X_{v(t)}^{i}\right] \cong X_{v(t+\tau)}^{i} \quad \text { for } \quad i=s, 0, u,
\end{gathered}
$$

and all $\tau \geqq 0$, and there are constants $c, d, v>0$ such that

$$
\begin{aligned}
& \left|\left(T_{v(t)} F(\tau)\right)(y)\right| \leqq c e^{-v \tau}|y| \text { for all } \tau \geqq 0 \text { and } y \in X_{v(t)}^{s}, \\
& \left|\left(T_{v(t)} F(\tau)\right)(y)\right| \geqq d e^{v \tau}|y| \text { for all } \tau \geqq 0 \text { and } y \in X_{v(t)}^{u} .
\end{aligned}
$$

We notice that the definition of a (weak) hyperbolic structure does not imply a splitting of the whole restricted tangent bundle of $X$ over $I(F)$. Moreover, the semiflow $F(t)$ has a (weak) hyperbolic structure for $I(F)$ precisely if it has a (weak) hyperbolic structure for $\bigcap_{t \geqq 0} F(t)[I(F)]$. In case that the (weak) hyperbolic set is equal to $A(F)$ and $A(F)$ is compact, we call $F(t)$ an Anosov semiflow.

We will need the following two lemmas.

Lemma 4. Let $F(t)$ be a $C^{1}$-semiflow of $X$ and assume that $F(t)$ has a (weak) hyperbolic structure for an $\omega$-invariant set $I(F)$. Then $\widetilde{F(t)}$ has a (weak) hyperbolic structure for $\widetilde{I(F)}$.

Proof. For $v \in \widetilde{I(F)}$ we can write $T_{v} C(\mathbb{R}, X)=C(\mathbb{R}, X)$ and define

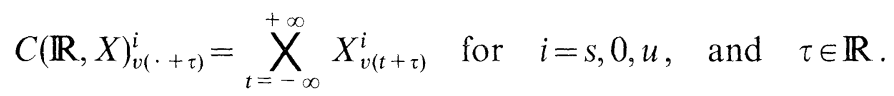

Since $F(t)$ has a (weak) hyperbolic structure for $I(F)$, it follows that there exists a continuous splitting of $\bigcup_{t=-\infty}^{+\infty} T_{v(\cdot+\tau)} C(\mathbb{R}, X)$ into a direct sum $C(\mathbb{R}, X)^{s} \oplus C(\mathbb{R}, X)^{0} \oplus C(\mathbb{R}, X)^{u}$, where

$$
C(\mathbb{R}, X)^{i}=\bigcup_{\tau=-\infty}^{+\infty} C(\mathbb{R}, X)_{v(\cdot+\tau)}^{i} \quad \text { for } \quad i=s, 0, u,
$$

$$
T_{v(\cdot+\tau)} \widehat{F(t)}\left[C(\mathbb{R}, X)_{v(\cdot+\tau)}^{i}\right] \cong C(\mathbb{R}, X)_{v(\cdot+t+\tau)}^{i} \quad \text { for } \quad i=s, 0, u, \quad \text { and } \quad \tau \in \mathbb{R} \text {. }
$$


Moreover, we have

$$
\begin{aligned}
\left\|T_{v} \widetilde{F(\tau)}(w)\right\| & =\int_{-\infty}^{+\infty} e^{-|t|}\left|T_{v(t)} F(\tau)(w(t))\right| d t \\
& \leqq c e^{-v \tau} \int_{-\infty}^{+\infty} e^{-|t|}|w(t)| d t \\
& =c e^{-v \tau}\|w\|
\end{aligned}
$$

for all $\tau \geqq 0$ and $w \in C(\mathbb{R}, X)_{v}^{s}$, and analogously

$$
\left\|T_{v} \widetilde{F(\tau)}(w)\right\| \geqq d e^{v \tau}\|w\|
$$

for all $\tau \geqq 0$ and $w \in C(\mathbb{R}, X)_{v}^{u}$.

Lemma 5. Let $F(t)$ be a $C^{1}$-semiflow of $X$ and $I(F)$ an $\omega$-invariant subset of $F(t)$. If $\bigcap_{t \geq 0} F(t)[I(F)]$ is compact in $X$, then $\widetilde{I(F)}$ is compact in $C(\mathbb{R}, X)$.

Proof. As in Proposition 1 we obtain that $p_{\tau}[\widetilde{I(F)}]=\bigcap_{t \geqq 0} F(t)[I(F)]$ for all $\tau \in \mathbb{R}$. Thus we can write

$$
\widetilde{I(F)}=\left\{v \in C\left(\mathbb{R}, \bigcap_{t \geqq 0} F(t)[I(F)]\right) \mid F(v(t))=v(t+1) \text { for all } t \in \mathbb{R}\right\} .
$$

The assertion then follows from Tychonov's theorem, since $\widetilde{I(F)}$ is complete.

We can now prove the inverse limit stability of compact (weak) hyperbolic invariant sets.

Theorem 2. Let $F(t)$ be a $C^{1}$-semiflow of $X$, and $I(F)$ an $\omega$-invariant set for $F(t)$. We assume that $F(t)$ has a (weak) hyperbolic structure for $I(F)$ and that $\bigcap_{t \geqq 0} F(t)[I(F)]$ is compact in $X$. Then there exists a $C^{1}$-neighborhood $U$ of $F(t)$ in $\operatorname{Sem}^{t \geq 0}(X)$ such that for all $G(t) \in U$ there is an $\omega$-invariant set $J(G)$ for $G(t)$ such that there exists a homeomorphism $H: \overparen{I(F)} \rightarrow \widehat{J(G)}$ and a function $\alpha: \overparen{I(F)} \rightarrow] 0, \infty[$ with $H \widetilde{F(t)}(v)$ $=\widetilde{G(\alpha(v) t)} H(v)$ for all $v \in I(F)$ and $t \in \mathbb{R}$. By choosing the neighborhood $U$ sufficiently small, we can get $H$ arbitrarily close to the identity, and the number $\alpha(v)$ for $v \in \widetilde{I(F)}$ arbitrarily close to the number 1 . Subject to these restrictions, the maps $H$ and $\alpha$ are unique.

Proof. The proof follows Robinson [10], and is based on the implicit function theorem. We first try to find a conjugacy for the time one maps, i.e., we are looking for continuous functions $H: \widetilde{I(F)} \rightarrow C(\mathbb{R}, X)$ and $\alpha: \widetilde{I(F)} \rightarrow] 0, \infty[$ such that

$$
H \tilde{F}(v)=\widetilde{G(\alpha(v))} H(v) \text { for all } v \in \widetilde{I(F)} .
$$

Setting $H=\exp (T)$, we can rewrite Eq. (4) as

$$
\left.\exp ^{-1} \widetilde{(G(\alpha(v))} \exp (T)^{\tilde{F}^{-1}}(v)\right)-T(v)=0 \text { for all } v \in \widetilde{I(F)} \text {. }
$$

As in [10, p. 173] it follows from the implicit function theorem that Eq. (5) has unique solutions $T$ and $\alpha$, if $G(t)$ is sufficiently $C^{1}$-close to $F(t)$, and hence $\overline{G(t)}$ is sufficiently $C^{1}$-close to $\widetilde{F(t)}$ with respect to the corresponding quasinorm on $\operatorname{Sem}^{1}(C(\mathbb{R}, X))$. Since $\widetilde{F(t)}$ has a (weak) hyperbolic structure for $\widetilde{I(F)}$ by Lemma 4 , we obtain that $\widetilde{F(t)_{\mid I I F)}}$ is expansive, i.e., for every $\varepsilon>0$ there exists a $\delta>0$ such that, ir $v, w \in \widetilde{I(F)}$ and 
$g: \mathbb{R} \rightarrow \mathbb{R}$ is continuous with $g(0)=0$ and $\|\widetilde{F(t)}(v)-\widetilde{F(g(t))}(w)\| \leqq \delta$ for all $t \in \mathbb{R}$, then $w=\widetilde{F(t)}(v)$ for all $|t| \leqq \varepsilon$. This is a local result and follows from the fact that, for $v \in \widetilde{I(F)}$ and a sufficiently small constant $\varepsilon>0$, the sets

$$
\begin{gathered}
W_{\tilde{F}}^{s, \varepsilon}(v)=\{w \in S(\widetilde{F}) \mid\|\widetilde{F(-t)}(v)-\widetilde{F(-t)}(w)\| \leqq \varepsilon \text { for all } t \geqq 0\}, \\
W_{\widetilde{F}}^{u, \varepsilon}(v)=\{w \in S(\widetilde{F}) \mid\|\widetilde{F(t)}(v)-\widetilde{F(t)}(w)\| \leqq \varepsilon \text { for all } t \geqq 0\},
\end{gathered}
$$

intersect in $\{v\}$. Expansiveness implies that $H$ is one-to-one, provided the $C^{0}$-distance from $H$ to the identity on $\widetilde{I(F)}$ is less than $\frac{1}{2} \varepsilon$. Namely, let $v, w \in \widetilde{I(F)}$ and suppose that $H(v)=H(w)$. Then

$$
\begin{aligned}
H \widetilde{F(t)}(v) & =\widetilde{G(\alpha(v) t)} H(v)=\widetilde{G(\alpha(v) t)} H(w) \\
& =\widetilde{G(\alpha(w) g(t))} H(w) H \widetilde{F(g(t))}(w)
\end{aligned}
$$

for all $t \in \mathbb{R}$, and thus

$$
\begin{gathered}
\|\widetilde{F(t)}(v)-\widetilde{F(g(t))} w\| \\
\leqq \\
\|\widetilde{F(t)}(v)-H \widetilde{F(t)}(v)\|+\|H \widetilde{F(t)}(v)-H \widetilde{F(g(t))}(w)\| \\
+\|H \widetilde{F(g(t))}(w)-\widetilde{F(g(t))}(w)\| \leqq \frac{1}{2} \varepsilon+0+\frac{1}{2} \varepsilon=\varepsilon
\end{gathered}
$$

for all $t \in \mathbb{R}$, which implies that $v=w$. Since $\widetilde{I(F)}$ is compact in $C(\mathbb{R}, X)$ by Lemma 5, it follows that $H: \widetilde{I(F)} \rightarrow H[\widetilde{I(F)}]$ is a homeomorphism. To see that its range is contained in $S(\widetilde{G})$, we use again Lemmas 1 and 2 . They show that the "adjusted" map $\hat{H}$ also commutes $\widetilde{F(t)}$ and $\widetilde{G(t)}$ on $\widetilde{I(F)}$ and satisfies $\hat{H}[\widetilde{I(\widetilde{F})}] \subseteq S(\widetilde{G})$. Uniqueness then implies that $H=\hat{H}$. We therefore may choose $J(G)=p_{0}[H[\widehat{I(F)}]]$.

Now let $t \in \mathbb{R}$ and $v \in \widetilde{I(F)}$. Then

and

$$
\begin{aligned}
\widetilde{G(\alpha(v))} \overline{(G(\alpha(v) t)} H \widetilde{F(-t))}(v) & =\widetilde{G(\alpha(v) t)} \overline{G(\alpha(v))} H \overline{F(-t)}(v) \\
& =\widetilde{G(\alpha(v) t)} H \widetilde{F(1)} \widetilde{F(-t)}(v) \\
& =\widetilde{(G(\alpha(v) t)} H \widetilde{F(-t))} \overline{F(1)}(v),
\end{aligned}
$$

$\widetilde{(G(\alpha(v) t)} H \widetilde{F(-t)}-\mathrm{id})(v)=(\widetilde{G(\alpha(v)} t)-\widetilde{F(t)}) H \widetilde{F(-t)}+\widetilde{F(t)}(H-\mathrm{id}) \widetilde{F(-t))}(v)$,

which shows that $\widehat{G(\alpha(v) t)} H \overparen{F(-t)}$ is close to the identity. It follows therefore from uniqueness that

$$
H \widetilde{F(t)}(v)=\widetilde{G(\alpha(v) t)} H(v) .
$$

This completes the proof of Theorem 2 .

Let us now make the following observations.

First, it is not difficult to see that $G(t)$ has a (weak) hyperbolic structure for $J(G)$, because the set of hyperbolic linear maps of $X$ is open in the set of continuous linear maps of $X$.

Secondly, if $I(F)$ is equal to $A(F)$, i.e., $I(F)$ is the maximal compact invariant set for $F(t)$, then the range of the above conjugacy will be all of $S(\widetilde{G})$.

This leads to the following result:

Theorem 3. Anosov semiflows of $X$ are $C^{1}$-inverse limit stable and form an open subset in $\operatorname{Sem}^{1}(X)$. 


\section{Axiom A Semiflows}

We end this paper with a conjecture about a general stability theorem for semiflows. Given a $C^{r}$-semiflow $F(t)$ of $X, r \geqq 1$, and $v \in S(\widetilde{F})$, we define stable and unstable sets of $F(t)$ at $v$ through

$$
\begin{aligned}
& W_{F}^{s}(v)=\{w \in S(\tilde{F})\|\widetilde{F(t)}(v)-\widetilde{F(t)}(w)\| \underset{t \rightarrow+\infty}{\longrightarrow} 0\}, \\
& W_{F}^{u}(v)=\{w \in S(\widetilde{F})\|\widetilde{F(t)}(v)-\widetilde{F(t)}(w)\| \underset{t \rightarrow-\infty}{\longrightarrow} 0\} .
\end{aligned}
$$

These sets contain the global solutions of $F(t)$ which approach and leave $v$ as $t$ goes to $+\infty$ and $-\infty$, respectively. In case that the linearized semiflow at $v$ is hyperbolic, they should be injectively $C^{r}$-immersed submanifolds of $C(\mathbb{R}, X)$.

Moreover, we define

$$
\begin{array}{r}
\Omega(F)=\{x \in X \mid \text { for every neighborhood } U \text { of } x \text { in } X \text { there } \\
\text { exists a } t \geqq 0 \text { such that } F(t)[U] \cap U \neq \emptyset\},
\end{array}
$$

that is the set of nonwandering points of $F(t)$.

We say that $F(t)$ satisfies Axiom $A$ (compare Smale [11]) if

1) $F(t)$ has a (weak) hyperbolic structure for $\Omega(F)$,

2) $\widehat{\Omega(F)}$ is the closure of the set of all periodic solutions of $F(t)$.

We conjecture that the following conditions are necessary and sufficient for a $C^{r}$-semiflow $F(t)$ of $X, r \geqq 1$, to be $C^{r}$-inverse limit stable:

1) $F(t)$ satisfies Axiom $A$,

2) the stable and unstable manifolds of the elements in $\widetilde{\Omega(F)}$ intersect transversally,

3) $S(\widetilde{F})$ is compact in $C(\mathbb{R}, X)$.

Let us indicate the main steps in a proof of the inverse limit stability from assumptions 1) to 3 ).

The set $S(\widetilde{F})$ is the (disjoint) union of the unstable manifolds of the elements in $\overline{\Omega(F)}$. This is a consequence of the compactness of $S(\widetilde{F})$. Since $F(t)$ has a (weak) hyperbolic structure for $\Omega(F)$, it follows from Theorem 2 that there exists an $\omega$-invariant set $J(G)$ for $G(t)$ and a homeomorphism $H: \overline{\Omega(F)} \rightarrow \overline{J(G)}$ and a function $\alpha: \overline{\Omega(F)} \rightarrow] 0, \infty[$ such that the following diagram commutes for all $t \geqq 0$ :

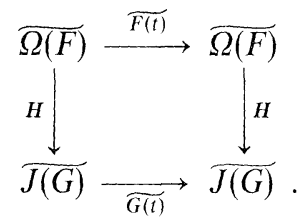

Moreover, $G(t)$ has a (weak) hyperbolic structure for $J(G)$. In order to see that $J(G)$ is equal to $\Omega(G)$, we observe that $H[\widehat{Q(F)}] \cong \widetilde{\Omega(G)}$, because the periodic solutions of $F(t)$ are dense in $\widetilde{\Omega(F)}$. Also, there exists a spectral decomposition of $\Omega(G)$ into finitely many basic sets with corresponding filtrations (see [11] for the definitions). This implies that $\Omega(G)$ is contained in a small neighborhood of the basic sets, and the proof of the opposite inclusion reduces to a study near these basic sets. Arguments similar to those used in the flow case should apply here, too. 
Next we observe that the set $S(\widetilde{G})$ is the disjoint union of the unstable manifolds of the elements in $\widetilde{\Omega(G)}$, and using an observation in [8], we can construct local conjugacies between the unstable manifolds for each corresponding pair of elements in $\widetilde{Q(F)}$ and $\widetilde{\Omega(G)}$. These local conjugacies can then be extended to the global unstable manifolds by following along the global solutions. Combining all these conjugacies yields a commuting map between $S(\widetilde{F})$ and $S(\widetilde{G})$. What remains to show is the continuity, and here the transversality condition comes in.

Acknowledgement. I would like to thank Natalia Sternberg for her help.

\section{References}

1. Anosov, D.: Geodesic flows on compact Riemannian manifolds of negative curvature. Tr. Mat. Inst. Steklova 90 (1967) [English: Am. Math. Soc. translation (1969)]

2. Irwin, M.C.: Smooth dynamical systems. New York: Academic Press 1980

3. Komura, M.: Expansive properties of Lorenz attractors. In: The theory of dynamical systems and its applications to nonlinear problems, pp.4-26. Singapore: World Scientific 1984

4. Mané, R.: Hyperbolic sets for semilinear parabolic equations. Bol. Soc. Bras. Mat. 6, 145-153 (1975)

5. Moser, J.: On a theorem of Anosov. J. Differ. Equations 5, 411-440 (1969)

6. Palis, J.: On the local structure of hyperbolic points in Banach spaces. Ann. Acad. Bras. Cienc. 40, 263-266 (1968)

7. Pugh, C.C.: On a theorem of P. Hartman. Am. J. Math. 91, 363-367 (1969)

8. Quandt, J.: On the Hartman-Grobman theorem for maps. J. Differ. Equations 64, 154-164 (1986)

9. Quandt, J.: Stability of Anosov maps. Proc. Am. Math. Soc. (to appear)

10. Robinson, C.: Structural stability of vector fields. Ann. Math. 99, 154-175 (1974)

11. Smale, S.: Differentiable dynamical systems. Bull. Am. Math. Soc. 73, 747- 817 (1967)

Communicated by J.-P. Eckmann

Received June 22, 1987; in revised form October 29, 1987 